\title{
Enzymatic Reconstitution and Biosynthetic Investigation of the Bacterial Carbazole Neocarazostatin A
}

\author{
Yating Liu, ${ }^{\dagger} \bigcirc \mathrm{Li} \mathrm{Su},{ }^{\dagger, \bigcirc}$ Qing Fang, Jioji Tabudravu, ${ }^{\S \odot}$ Xiaohui Yang," Kirstie Rickaby,
}

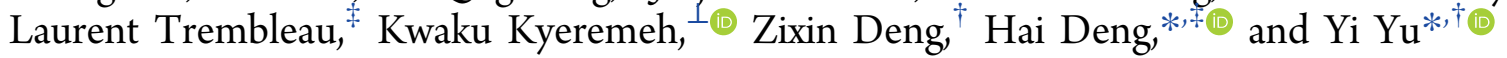

${ }^{\dagger}$ Institute of TCM and Natural Products, Key Laboratory of Combinatorial Biosynthesis and Drug Discovery (Ministry of Education), School of Pharmaceutical Sciences, Wuhan University, 185 East Lake Road, Wuhan 430071, People’s Republic of China ${ }^{\ddagger}$ Marine Biodiscovery Centre, Department of Chemistry, University of Aberdeen, Aberdeen, Scotland AB24 3UE, United Kingdom

${ }^{\S}$ School of Forensic \& Applied Sciences, Faculty of Science \& Technology, University of Central Lancashire, Preston, Lancashire, England PR1 2HE, United Kingdom

"School of Chemistry, Xiamen University, Xiamen 361005, People's Republic of China

${ }^{\perp}$ Department of Chemistry, University of Ghana, P.O. Box LG56, Legon-Accra, Ghana

\section{Supporting Information}

ABSTRACT: Tricyclic carbazole is an important scaffold in many naturally occurring metabolites, as well as valuable building blocks. Here we report the reconstitution of the ring A formation of the bacterial neocarazostatin A carbazole metabolite. We provide evidence of the involvement of two unusual aromatic polyketide proteins. This finding suggests how new enzymatic activities can be recruited to specific pathways to expand biosynthetic capacities. Finally, we leveraged our bioinformatics survey to identify the untapped capacity of carbazole biosynthesis.

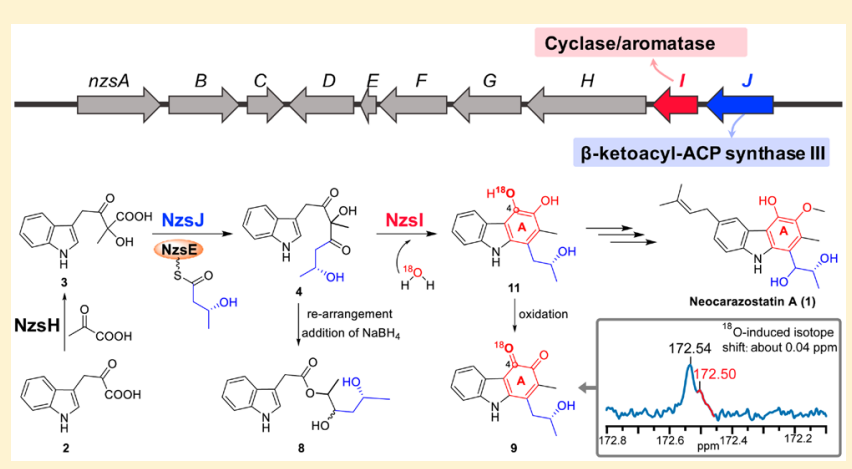

as butylhydroxytoluene and the drug flunarizine. ${ }^{2}$ There have been considerable interests among medicinal chemists to develop 1 due to its pharmacological potential. Neocarazostatin A 1 belongs to a group of simple carbazole alkaloids (CAs) with aliphatic side chains. Considering the oxidative status of ring $A$, this group of CAs can be categorized into four subgroups: indole-fused dihydroxyl-type CAs including $\mathbf{1}$ as a representative, indole-fused monohydroxyltype CAs such as carazostatin, indole-fused ortho-quinone CAs such as carquinonstatin $\mathrm{A}$, and nonaromatic type CA (Figure 1).

In our previous studies, we have delineated the functions of five key enzymes encoded in the biosynthetic gene cluster (BGC) of 1 (nzs), including the phytoene-synthase-like prenyltransferase NzsG and the P450 hydroxylase NzsA, ${ }^{1}$ a thiamine diphosphate-dependent enzyme $\mathrm{NzsH}^{3}$ a freestanding acyl carrier protein (ACP) NzsE and a classical $\beta$ ketoacyl-acyl carrier protein synthase III NzsF. ${ }^{4}$

Here, we demonstrated that in vitro reconstitution of NzsJ and I, together with $\mathrm{NzsH}$ and other necessary substrates and cofactors, enables the formation of the A ring of 1 , which is spontaneously oxidized into ortho quinone-containing carba-

Received: October 4, 2019

Published: November 15, 2019 
zole, a similar observation discovered by Kobayashi and coworkers in parallel to our study. ${ }^{5}$ Isotopic labeling studies demonstrate that one of the oxygens in the A ring is derived from water. Moreover, comparative genomics and a global network analysis of sequence similarity of $n z s H, J$, and $I$ suggested that these gene homologues are clustered, and the occurrence of these BGCs in the bacterial kingdom suggests important biological functions in these organisms.

Our previous studies suggested that both $n z s J$ and nzsI genes are essential for the production of 1 , and no obvious intermediates were accumulated in both $n z s J$ and $I$ knockout mutants. ${ }^{1}$ Bioinformatics analysis suggested that NzsJ is a putative FabH-like 3-ketoacyl-ACP synthase (KAS) III and that NzsI is a structural homologue of aromatase/cyclases (ARO/CYCs) that involve type II aromatic polyketide (PKS II) biosynthesis, strongly suggesting that Streptomyces sp. MA37 recruits new enzyme activities from the PKS II assembly line to the $\mathrm{CA}$ biosynthetic gene clusters to enable the evolution of biosynthetic capacity. During the manuscript preparation, reconstitution of the biosynthetic pathway of carquinostatin A, a structural homologue of 1 , was reported ${ }^{5}$ with the structural insights of one of the key biosynthetic enzymes, CqsB2, the homologue of NzsI with high sequence identity (80\%).

In this study, we provide a full account of our work and show that NzsJ and NzsI catalyze the formation of the A ring of 1. To this end, we set out in vitro enzymatic assays. Overexpression of NzsJ and NzsI in S. lividans allowed us to isolate and purify the recombinant protein to near homogeneity with the estimated molecular weight of $35 \mathrm{kDa}$ and 28 $\mathrm{kDa}$, respectively, as determined by SDS-PAGE analysis (Figure S1). Considering the instability of $\alpha$-hydroxyl acyloin 3 from $\mathrm{NzsH}$ (the structure of 3 has been revised here compared to our previous structural interpretation ${ }^{3}$ ), we first performed a one-pot reaction by incubating NzsJ $(1 \mu \mathrm{M})$ with the enzymatic systems of NzsH and NzsE we've established before to generate the acyloin and 3-hydroxybutyryl-NzsE in situ, respectively; two new compounds with mass-to-charge ratios $(m / z)$ of 290.1382 were formed as observed in the LCHR-ESIMS analysis. N-Acetylcysteamine (SNAC) is commonly used as a simplified synthetic mimic of the reactive biosynthetic intermediates, such as acyl-ACP in the biosynthetic studies. ${ }^{6,7}$ To isolate new compounds for structural elucidation, we chemically prepared $R$-3-hydroxybutyryl-SNAC (Figures S2-4). Upon incubation of NzsJ with R-3hydroxybutyryl-SNAC and the enzymatic system of $\mathrm{NzsH}$, the target molecules with identical molecular weights and UV absorption but different retention times (compound 5 and 6) were observed in the HR-ESIMS and HPLC analyses (Figure 2A, Figure S5). Interpretation of NMR spectral and HRESIMS data suggested that $\mathbf{5}$ is likely to be an indole-acetyl ester despite the presence of the impurity (Figures S6-8). Compound $\mathbf{6}$ was proposed to be the isomer of 5 .

To further confirm the structures of $\mathbf{5}$ and $\mathbf{6}$, we added $\mathrm{NaBH}_{4}$ into the enzymatic mixture at the end of the assay, resulting in the appearance of two new ions, 7 and 8, with both of which have $m / z$ of 292.1540 as observed in the HR-ESIMS (Figure S9), further confirming the presence of only one ketone functional group. HR-ESLMS data for compounds 7 and 8 indicated a molecular formula of $\mathrm{C}_{16} \mathrm{H}_{21} \mathrm{NO}_{4}$, suggesting 7 degrees of unsaturation. The structure of $\mathbf{8}$ was proposed to be the reduced product of 5 by the interpretation of NMR spectral data (Figures S10-13, Table S1) coupled with

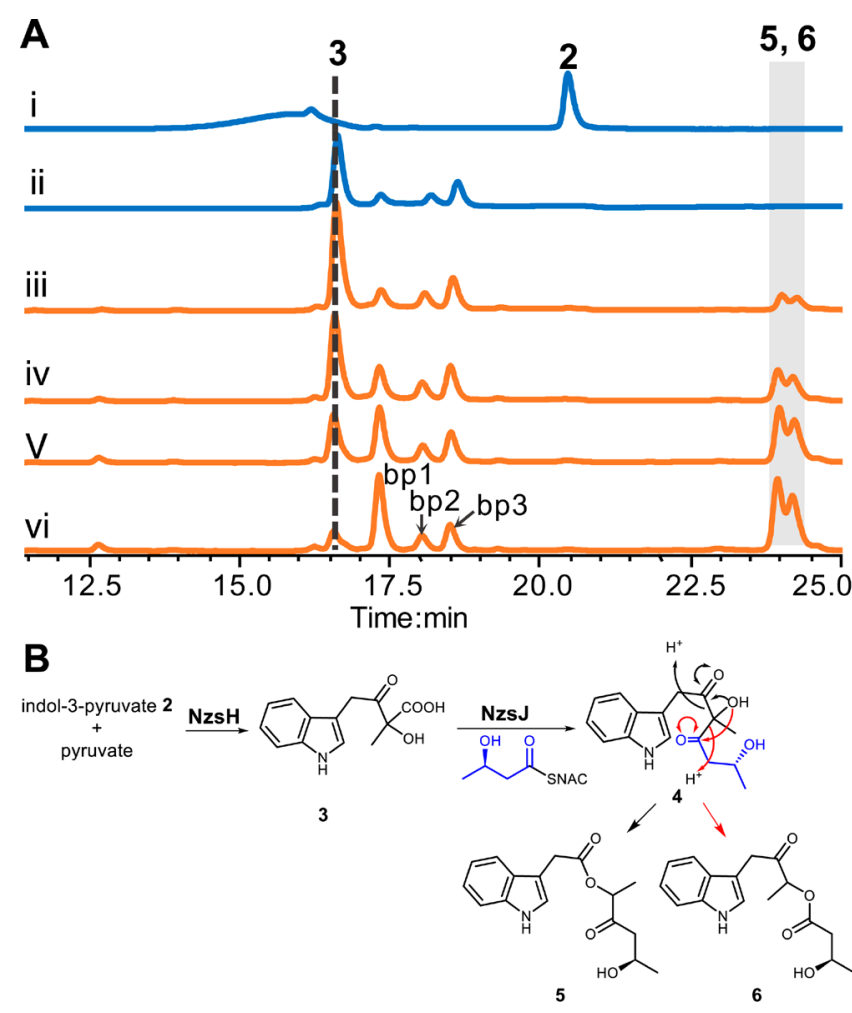

Figure 2. Functional dissection of NzsJ. (A) HPLC analysis of the one-pot enzymatic reaction catalyzed by $\mathrm{NzsH} / \mathrm{J}$. (i) Control reaction using pyruvate, indol-3-pyruvate (2), and boiled $\mathrm{NzsH} / \mathrm{J}$; (ii) control reaction using pyruvate, 2, 3-hydroxybutyryl-SNAC, and boiled NzsJ; (iii-vi) time course of the $\mathrm{NzsH} / \mathrm{J}$ one-pot reactions containing pyruvate, 2, 3-hydroxybutyryl-SNAC, NzsH, and NzsJ, terminated at $10,30,60$, and $120 \mathrm{~min}$, respectively. The UV absorption was monitored at $280 \mathrm{~nm}$. It is noteworthy that three HPLC peaks at the retention times of 17.5-18.5 min were putative byproducts (bp) during the biotransformation. The bp1 has the $\mathrm{m} / z$ of 378.1576 as observed in HR-ESIMS. However, we were unable to determine the $m / z$ of bp2 and bp3. (B) The proposed reaction of NzsJ.

theoretical calculations (Figures S14-16). Compound 7 was deduced to be the isomer of $\mathbf{8}$.

Analysis of the structures of 5, 6 and their derivatives, 7 and $\mathbf{8}$, led to speculation that $\mathbf{5}$ or $\mathbf{6}$ may be the substrate of NzsI. Surprisingly, an enzymatic assay of NzsI with the isolated 5 and $\mathbf{6}$ resulted in no new product, strongly suggesting that 5 and $\mathbf{6}$ were rearranged enzymatic products, a similar observation of recent parallel report. ${ }^{5}$

This led us to speculate that $\mathbf{4}$ is the bonafide product of NzsJ that underwent a spontaneous $\alpha$-ketol rearrangement reaction (Figure 2B). Such a rearrangement is also observed in the interconversion from the dibenzo[b]fluorene skeleton to a benzo $[g]$ chromene in the synthetic experiments ${ }^{8}$ and the biogenetic interconversions between prekinamycin and isoprekinamycin ${ }^{9}$ (Figure S17). Density functional theory (DFT) calculations also confirmed that the structure of $\mathbf{4}$ has a higher energy than that of $\mathbf{5}$ or $\mathbf{6}$ (Table S2). It is noteworthy that no ions with a $4 \mathrm{Da}$ increase were observed, strongly suggesting that the predicted intermediate $\mathbf{4}$ was not accumulated and underwent immediate rearrangement.

To investigate the roles of both NzsJ and NzsI, we performed a one-pot reaction of NzsI in the presence of NzsJ, 3-hydroxybutyryl-SNAC, and the NzsH system. A new product (9) was generated with $m / z$ of 270.1125 , as observed 
in the HR-ESIMS analysis (Figure 3A, Figure S18). In a control assay with boiled NzsI and in other control assays lacking either the NzsH system or NzsJ or SNAC substrate, the formation of 9 was not observed.

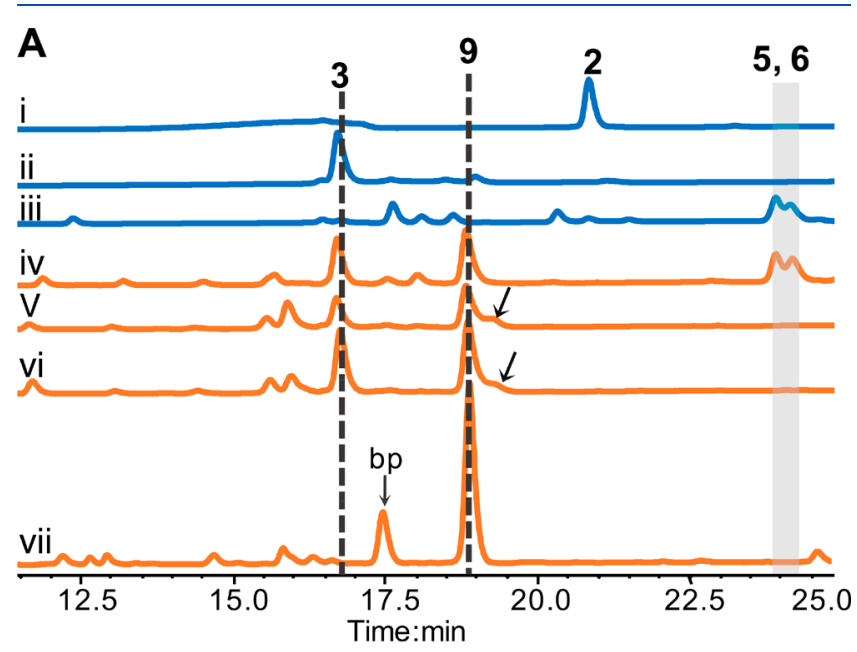

B

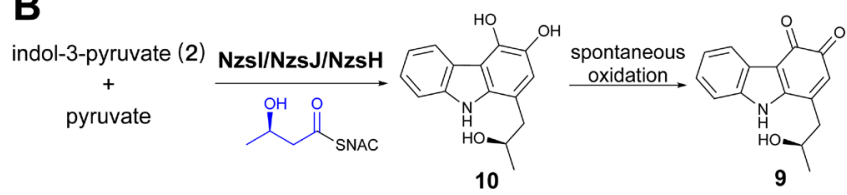

Figure 3. Functional dissection of NzsI. (A) HPLC analysis of the one-pot enzymatic reactions catalyzed by $\mathrm{NzsH} / \mathrm{I} / \mathrm{J}$. (i) Control reaction using pyruvate, $\mathbf{2}$, and boiled $\mathrm{NzsH} / \mathrm{I} / \mathrm{J}$; (ii) control reaction using pyruvate, 2, 3-hydroxybutyryl-SNAC, NzsH, and boiled NzsJ/I; (iii) $\mathrm{NzsH} / \mathrm{J}$ one-pot reactions plus boiled NzsI for $30 \mathrm{~min}$ of incubation. (iv) NzsI was added into reaction iii and incubated for another $30 \mathrm{~min}$; (v-vii) time course of one-pot reactions containing $\mathrm{NzsH} / \mathrm{I} / \mathrm{J}$, pyruvate, 2, and 3-hydroxybutyryl-SNAC, terminated at 30,60 , and $120 \mathrm{~min}$, respectively. The tiny peak following 9 corresponds to a tautomer (10 or 10a). The UV absorption was monitored at $280 \mathrm{~nm}$. (B) The proposed reaction of NzsI.

To confirm the structure of $\mathbf{9}$, a large-scale biotransformation reaction was carried out to afford the isolation of 9 (8 $\mathrm{mg}$ ). The inspection of $1 \mathrm{D}$ and $2 \mathrm{D}$ NMR spectral data demonstrated that 9 is an ortho-quinone carbazole (Figures S19-22). Intriguingly, bioinformatics analysis suggested that NzsI is not an oxidative enzyme, leading us to speculate $\mathbf{1 0}$ is the bona fide enzymatic product of NzsI but was oxidized spontaneously (Figure S23A). The presence of dithiothreitol (DTT, $1 \mathrm{mM}$ ) in the biotransformation of $\mathrm{NzsH} / \mathrm{J} / \mathrm{I}$ indeed extended the existence of the ortho-dihydroxyl compounds $\mathbf{1 0}$ as observed in HPLC and HR-MS analyses (Figure S23B,C).

This led us to propose that NzsI may install the hydroxyl group at the $\mathrm{C} 4$ of $\mathbf{4}$ from one water molecule. To verify this hypothesis, we carried out the biotransformation using isotopically enriched water. An assay of NzsI with the substrates in the buffer system enriched with $\mathrm{H}_{2}{ }^{18} \mathrm{O}(15 \%)$ was performed. Indeed, isotopically enriched 9a $\left([\mathrm{M}+2]^{+}\right.$: $18 \%$ ) was observed (Figure 4A,B), as determined by HRESIMS analysis and by comparison with the MS pattern of 9 (Figure S24). To further confirm that the hydroxyl group is indeed located at the $\mathrm{C} 4$ position in the $\mathrm{A}$ ring, a large-scale biotransformation reaction was performed to afford the isolation the mixture of 9 and isotopically enriched 9a. The inspection of ${ }^{13} \mathrm{C}\left\{{ }^{1} \mathrm{H}\right\}$ NMR of the resultant product
A

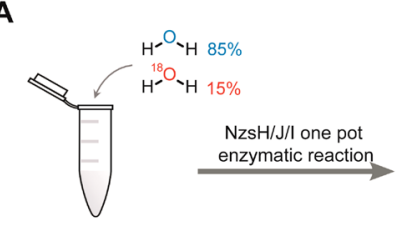

B
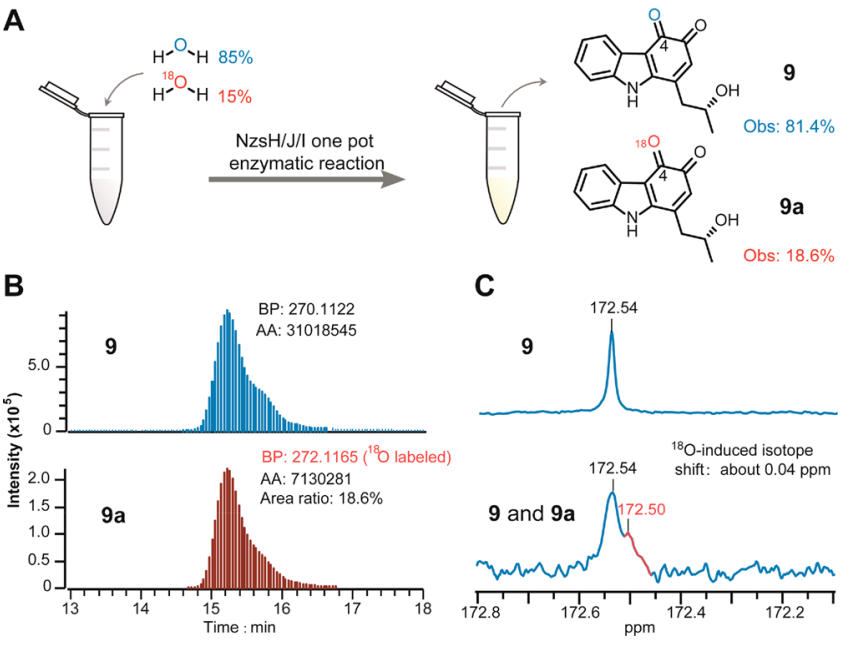

C

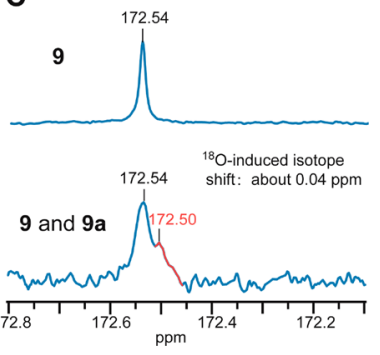

Figure 4. Isotope-labeling strategy and the representative mass chromatograms and NMR spectra. (A) Schematic depiction of the $\mathrm{H}_{2}{ }^{18} \mathrm{O}$ isotope substitution. (B) LC-HR-ESIMS analysis of the enzymatic mixture of the $\mathrm{NzsH} / \mathrm{I} / \mathrm{J}$ one-pot reaction. Extracted ion chromatograms representing the off-labeled 9 and on-labeled 9a. BP. base peak mass. AA: peak area. (C) ${ }^{18} \mathrm{O}$ causes an upfield shift $(0.04$ ppm) of attached C4 NMR signals in compound 9a.

demonstrated the ${ }^{18} \mathrm{O}$-induced isotope shifts at the ketone of C4 of 9 (Figure 4C, Figure S25). The magnitude of this isotope shift is $0.04 \mathrm{ppm}$ for the $\mathrm{C} 4$ attached to ${ }^{18} \mathrm{O}$, consistent with the previous observation. ${ }^{10}$

Taken together, these experiments confirmed that NzsI utilizes the acid-base chemistry to catalyze the cyclization, followed by nucleophilic aromatization of water to afford the catechol moiety of carbazole metabolites, strongly suggesting that NzsI is a new type of cyclases/aromatases.

Based on the oxygen pattern in the A ring, this group of bacterial carbazole metabolites can be categorized into four subgroups: monohydroxyl type, dihydroxyl type, orthoquinone type, and nonaromatic type (Figure S26). We propose that the A ring of the carbazole is assembled by NzsH, NzsJ, and NzsI or corresponding enzyme homologues. The assembly of the A ring is initiated by the carboligation of $\mathbf{2}$ and pyruvate in a reaction catalyzed by $\mathrm{NzsH}$ or its homologues to afford the corresponding $\alpha$-keto acid 3. Depending on the bioavailability of the Acyl-CoA species in the producing strains, an unidentified acyltransferase will catalyze the transthiolation reaction to load Acyl-CoA into NzsE or its homologues to generate acyl-tethered thioesters. NzsJ or its homologues will utilize both 3 and acyl-tethered thioesters to mediate the decarboxylation-driven retro-aldol reaction to generate the intermediate, followed by cyclization via abstraction of the acidic proton at the $\mathrm{C} 4$ position, spontaneous dehydration, and water-based nucleophilic aromatization to yield the catechol motif. Methylation or transamination followed by acylation would result in dihydroxyl type carbazoles. Reduction on the key catechol-type intermediate will generate the nonaromatic type. Currently, only one metabolite without an aromatic feature in the A ring has been isolated from Streptomyces sp. BCC26924. ${ }^{11}$ If a reduction is involved in the NzsI-mediated reaction, the hydride-based nucleophilic aromatization would afford the monohydroxyl type of carbazole metabolites.

The presence of NzsI homologues in the bacterial kingdom was investigated. A blast search using NzsI as the query in the NCBI database allowed identification of a collection of NzsI- 
like homologues that share a high amino acid sequence identity $(43 \%-80 \%)$. The corresponding genes are found in the genome of microorganisms, including the Gram-positive actinomycetes, the well-studied S. cattleya, the Gram-negative bacteria including the thermophilic bacterium Legionella gratiana, the soil-dwelling myxobacterium Sorangium cellosum, and the cyanobacterium Scytonema tolypothrichoides (Figure 5,

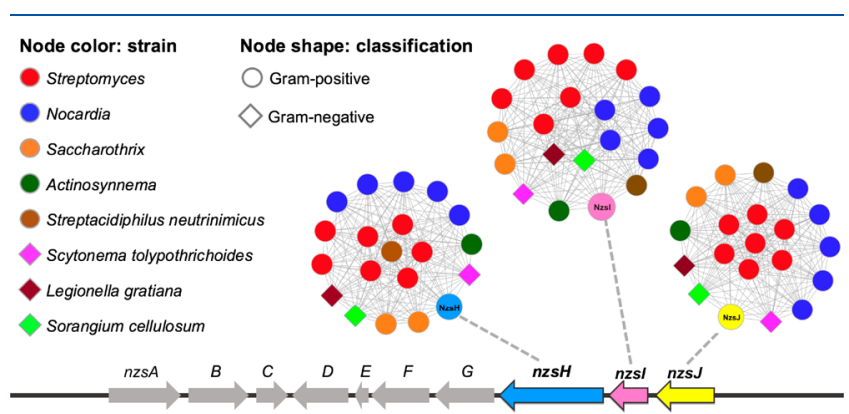

Figure 5. Sequence similarity network analysis of homologues of $\mathrm{NzsH}$, NzsI, and NzsJ. Each node in the network represents a homologous protein, and each edge represents the pairwise connection between two proteins with a blastP $E$ value $<1 \times 10^{-10}$.

Table S3). Strikingly, adjacent to these $n z s I$-like genes are $n z s H$-like and $n z s J$-like genes in some cases (Table S3). In silico analysis also indicated that these NzsJ-like open reading frames (ORFs) also share a high amino acid sequence identity (24\%$73 \%$ ) to NzsJ (Table S3). Furthermore, MEME prediction ${ }^{12}$ demonstrated that all of the NzsI-like ORFs share highly conserved motifs with NzsI (Figure S27), whereas all of the NzsJ-like ORFs share the conserved catalytic triad $\mathrm{C}-\mathrm{H}-\mathrm{N}$ with NzsJ and other KAS III proteins (Figure S28), suggesting that these ORFs should process the same chemical reactions as those for NzsI and NzsJ, respectively. However, analysis of the genes in the close proximity of these orfs suggested that these identified gene clusters encode completely different sets of auxiliary enzymes that were predicted to modify the CA ring system or the fatty acid chains. It is likely that these strains have the potential to produce not-yet-discovered new CA-like metabolites.

In conclusion, in vitro reconstitution of two unusual enzymes, NzsJ and NzsI, together with NzsH and other necessary substrates and cofactors, demonstrate the assembly of the A ring moiety of the bacterial carbazole metabolite $\mathbf{1}$. Isotopic labeling studies demonstrated that the hydroxyl group at $\mathrm{C} 4$ position of the $\mathrm{A}$ ring is originated from one water molecule. Bioinformatics analysis further uncovered that the homologues of NzsI are widespread in bacteria associated with $\mathrm{NzsH}$ and NzsJ homologues, suggesting that carbazole-type metabolites are ubiquitous in the bacterial kingdom.

\section{EXPERIMENTAL SECTION}

General Materials and Methods. All chemicals and solvents were obtained from Sigma-Aldrich except where noted. Oligonucleotide primers were synthesized by GenScript (Nanjing, China). The DNA sequencing of PCR products and all constructive plasmids was performed by TSINGKE Biological Technology (Wuhan, China). Restriction endonucleases and T4 ligase were purchased from NEB. High-fidelity DNA polymerase KOD was purchased from TOYABO. A typical PCR reaction contained 10-100 ng of the DNA template, $0.5 \mu \mathrm{M}$ of each primer, $2 \mu \mathrm{L}$ of $10 \times \mathrm{KOD}$ buffer, and $0.3 \mu \mathrm{L}$ of highfidelity KOD polymerase. Thermocycling was carried out in Bio-Rad C1000 thermocycler. E. coli DH10B was used as a cloning host. E. coli
ET12567/pUZ8002 was used for intergeneric conjugation between $E$. coli and Streptomyces. E. coli DH10B, and E. coli ET12567/pUZ8002 were cultured in Luria-Bertani (LB, tryptone $1 \%$, yeast extract $0.5 \%$, $\mathrm{NaCl} 1 \%)$ or $\mathrm{LB}$ agar medium at $37^{\circ} \mathrm{C}$. S. lividans TK24 and $\mathrm{S}$. lividans 1326 were grown on MS agar plates (soybean $2 \%$, D-mannitol $2 \%$, agar $2 \%$ ) at $28{ }^{\circ} \mathrm{C}$ for sporulation and in the YEME medium (glucose $1 \%$, tryptone $0.5 \%$, yeast extract $0.3 \%$, malt extract $0.3 \%$, sucrose $10.3 \%$ ) for protein overexpression. The MS medium with additional $10 \mathrm{mM} \mathrm{MgCl} 2$ was used for intergeneric conjugation. HPLC analysis was carried out on a Shimadzu (Kyoto, Japan) HPLC instrument equipped with a degasser (DGU-20A3), an autosampler (SIL-20A), a column oven (CTO-20A) and two pumps (LC-20AT), and Phenomenex columns $(\mathrm{C} 18,5 \mu \mathrm{m}) 250 \mathrm{~mm} \times 4.6 \mathrm{~mm}$. HPLC conditions for analysis of the one-pot reaction: the $\mathrm{C} 18$ column was pre-equilibrated with $20 \% \mathrm{~B}$ and developed at a flow rate of $0.8 \mathrm{~mL} /$ min, $0-20 \mathrm{~min}$, a linear gradient from $80 \%$ A to $20 \% \mathrm{~A} ; 20-25 \mathrm{~min}$, a linear gradient from $80 \%$ A to5\% A; 25-27 min, constant with 5\% A; 27-30 min, a linear gradient to $80 \%$ A; 30-35 min, constant with $80 \%$ A; UV absorption was monitored at $280 \mathrm{~nm}$. Solvent A was $0.1 \%$ formic acid in $\mathrm{H}_{2} \mathrm{O}$, and solvent $\mathrm{B}$ was $0.1 \%$ formic acid in $\mathrm{CH}_{3} \mathrm{CN}$. HR-ESI-MS analysis was carried out in the positive ion mode by using a Thermo Scientific LTQ XL Orbitrap mass spectrometer equipped with a Thermo Scientific Accela 600 pump (Thermo Fisher Scientific Inc.). Each of the LC conditions were described as above. All MS analysis parameters were as follows: $45 \mathrm{~V}$ capillary voltage, $45{ }^{\circ} \mathrm{C}$ capillary temperature, auxiliary gas flow rate 10 arbitrary units, sheath gas flow rate 40 arbitrary units, $3.5 \mathrm{kV}$ spray voltage, and 50-1000 Amu mass range (maximum resolution 30000 ).

Chemical Synthesis of 3-Hydroxybutyryl-SNAC. 3-Hydroxybutyryl-SNAC was synthesized as a simplified mimic of the reactive biosynthetic intermediate, which can be recognized by NzsJ in vitro. (R)-(-)-3-Hydroxybutanoyl sodium salt $(630 \mathrm{mg}, 5 \mathrm{mmol})$ was added to a solution of $\mathrm{CF}_{3} \mathrm{SO}_{3} \mathrm{H}(750 \mathrm{mg}, 5 \mathrm{mmol})$ in $25 \mathrm{~mL}$ of dichloromethane (DCM), and the mixture was stirred at $0{ }^{\circ} \mathrm{C}$ for $1 \mathrm{~h}$. Then the $N$-acetylcysteamine (NAC, $500 \mathrm{mg}, 4.2 \mathrm{mmol}, 1$ equiv), $N$ (3-(dimethylamino)propyl)- $N^{\prime}$-ethylcarbodiimide hydrochloride (EDCI, $800 \mathrm{mg}, 1$ equiv), and $N, N$-dimethylpyridin-4-amine (DMAP, $52 \mathrm{mg}, 0.1$ equiv) were sequentially added. The reaction mixture was stirred at $22{ }^{\circ} \mathrm{C}$ for $16 \mathrm{~h}$. After removing the solid by filtration, the filtrate was poured into $10 \mathrm{~mL}$ of cold water and extracted with ethyl acetate. Then the ethyl acetate layer was concentrated to dryness. The residue was dissolved in $2 \mathrm{~mL} \mathrm{DCM}$, and the reaction was monitored by TLC $(\mathrm{DCM} /$ methanol $=15 / 1)$. The solvent was removed, and the residue was purified on a silica gel flash column $(\mathrm{DCM} /$ methanol $=100 / 1)$ to yield 3-hydroxybutyrylSNAC $(0.12 \mathrm{~g}, 12 \%):{ }^{1} \mathrm{H}$ NMR, $\delta_{\mathrm{H}}\left(\mathrm{CDCl}_{3}, 400 \mathrm{MHz}\right) 1.21(\mathrm{~d}, J=$ $6.3 \mathrm{~Hz}, 3 \mathrm{H}), 1.95(\mathrm{~s}, 3 \mathrm{H}), 2.70(\mathrm{~m}, 2 \mathrm{H}), 3.03(\mathrm{~m}, 2 \mathrm{H}), 3.43(\mathrm{~m}, 2 \mathrm{H})$, $4.24(\mathrm{~m}, 1 \mathrm{H}) ;{ }^{13} \mathrm{C}\left\{{ }^{1} \mathrm{H}\right\} \mathrm{NMR}, \delta_{\mathrm{C}}\left(101 \mathrm{MHz}, \mathrm{CDCl}_{3}\right) 199.6(\mathrm{CO}, \mathrm{C}-$ 7), 170.8 (CO, C-2), 65.3 (COH, C-9), 52.7 (CH2, C-8), 39.5 (CH2, C-4), 29.0 (CH2, C-5), 23.4 ( CH3, C-1), 22.9 (CH3, C-10); HRESIMS $m / z[\mathrm{M}+\mathrm{H}]{ }^{+}$calcd for $\mathrm{C}_{8} \mathrm{H}_{16} \mathrm{NO}_{3} \mathrm{~S}^{+}$206.0845, found 206.0843.

Construction of NzsJ and Nzsl Overexpression Plasmid. The genes $n z s J$ were amplified from $S$. sp. MA37 genomic DNA using the primer pair His-NzsJ-F and His-NzsJ-R (Table S4) by high-fidelity PCR. The PCR-amplified products were purified and then cloned into a NdeI/HindIII cleaved expression vector pWDYHS01 by using an InFusion HD cloning kit (Clontech) to yield pWDY900. pWDY900 was then transformed into S. lividans TK24, and the resulting colonies were selected by the aac(3)IV resistant marker and confirmed by PCR to obtain the recombinant strain WDY900. The strain WDY900 was used for overexpression of the His-tagged NzsJ in Streptomyces. The genes $n z s I$ were PCR amplified from MA37 genomic DNA using the primers His-NzsJ-F/R and His-NzsI-F/R (Table S5). The PCR products were purified from an agarose gel and then cloned into an $\mathrm{NdeI} /$ HindIII linearized vector, pWDYHS01, using an InFusion HD cloning kit (Clontech). The ligation mixture was transformed into competent E. coli $\mathrm{DH} 10 \mathrm{~B}$ cells by heat shock. The recovery culture was plated on LB agar containing apramaycin $(50 \mu \mathrm{g} / \mathrm{mL})$ to screen 
the positive clones, and then the positive assembly was confirmed by digestion and sequencing.

Expression and Purification of Nzsl and NzsJ. E. coli ET12567/pUZ8002 was first transformed with the verified plasmids and then conducted general intergeneric conjugation with the $S$. lividans TK24 or 1326 to yield the protein overexpressed strain WDY900 and WDY901 (Table S6), respectively. The overexpression strain WDY900 or WDY901 was inoculated into $50 \mathrm{~mL}$ of the TSBY medium supplemented with $50 \mu \mathrm{g} / \mathrm{mL}$ apramycin and grown at $28{ }^{\circ} \mathrm{C}$ with shaking at $200 \mathrm{rpm}$ for 2 days. The preculture was transferred to $500 \mathrm{~mL}$ of the YEME medium supplemented with $50 \mu \mathrm{g} / \mathrm{mL}$ apramycin at $28{ }^{\circ} \mathrm{C}$ with shaking at $200 \mathrm{rpm}$ for 2 days. Protein expression was induced with the addition of $0.5 \mathrm{mM}$ (final concentration) thiostrepton. After an additional $60 \mathrm{~h}$ of incubation, the cells were harvested by centrifugation at $5000 \mathrm{~g}$ for $20 \mathrm{~min}$ at $4{ }^{\circ} \mathrm{C}$. The cell pellets collected by centrifugation were resuspended in icecold lysis buffer $(20 \mathrm{mM}$ Tris- $\mathrm{HCl}, 300 \mathrm{mM} \mathrm{NaCl}, 10 \mathrm{mM}$ imidazole, and $10 \%(\mathrm{v} / \mathrm{v})$ glycerol, $\mathrm{pH} 8.0)$ and disrupted by a high-pressure homogenizer machine (Avestin, EmulsiFlex-C3). Cell debris was removed via centrifugation $\left(4{ }^{\circ} \mathrm{C}, 12000 \mathrm{~g}, 30 \mathrm{~min}\right)$, and the supernatant was filtered before loading onto a $5 \mathrm{~mL}$ HisTrapHP column (GE Healthcare). The HisTrapHP column was washed by six concentration-step elution buffers, with $15 \mathrm{~mL}$ for each step $(20 \mathrm{mM}$ Tris-HCl, $300 \mathrm{mM} \mathrm{NaCl}, 10 \%$ (v/v) glycerol, along with $20 \mathrm{mM}, 50$ $\mathrm{mM}, 100 \mathrm{mM}, 150 \mathrm{mM}, 200 \mathrm{mM}$, or $300 \mathrm{mM}$ imidazole, $\mathrm{pH} 8.0$ ). The elution fractions were checked by SDS-PAGE, and then the desired elution fractions were concentrated using centrifugal filter units (Milipore, 10000 MWCO, Merck). The concentrated protein solution was subsequently desalted using a PD-10 column (GE Healthcare) pre-equilibrated with the elution buffer $(20 \mathrm{mM}$ Tris$\mathrm{HCl}, 100 \mathrm{mM} \mathrm{NaCl}$, and $10 \%(\mathrm{v} / \mathrm{v})$ glycerol, $\mathrm{pH} \mathrm{8.0)}$. The desalted effluent was centrifuged again and was then aliquoted, flash-frozen in liquid nitrogen, and stored at $-80{ }^{\circ} \mathrm{C}$. SDS-PAGE and spectrophotometric analysis (Bradford assay kit, Promega, BSA used as standard, absorbance at $595 \mathrm{~nm}$ ) were used to check the purity and concentration of the protein.

In Vitro Enzymatic Assay of the NzsH/J One-Pot Reaction. The NzsH/J one-pot reaction was performed by incubating $1 \mu \mathrm{M}$ NzsJ and $1 \mathrm{mM}$ 3-hydroxybutyryl-SNAC with the enzyme systems of $\mathrm{NzsH}$. The reaction mixture was incubated at $30{ }^{\circ} \mathrm{C}$ for the scheduled time and quenched by an equal volume of ice-cold methanol. The clarified supernatant was obtained by centrifugation at $13000 \mathrm{~g}$ for 30 min and then subjected to HPLC and HR-ESIMS analysis. The NzsH and boiled $\mathrm{NzsH} / \mathrm{J}$ added mixture were used as control reactions.

In Vitro Enzymatic Assay of the $\mathrm{NzsH} / \mathrm{J} / \mathrm{I}$ One-Pot Reaction. The $\mathrm{NzsH} / \mathrm{J} / \mathrm{I}$ one-pot reaction was performed by adding $1 \mu \mathrm{M} \mathrm{NzsI}$ in the mixture of the $\mathrm{NzsH} / \mathrm{J}$ one-pot system, as described above. The mixture was incubated at $30{ }^{\circ} \mathrm{C}$ for the scheduled time and quenched by an equal volume of precooled $\mathrm{MeOH}$. The clarified supernatant was obtained by centrifugation at $13000 \mathrm{~g}$ for $30 \mathrm{~min}$ and then subjected to HPLC and HR-ESIMS analysis. The NzsH, boiled $\mathrm{NzsH} / \mathrm{J} / \mathrm{I}$ added, and $\mathrm{NzsH} / \mathrm{J}$ one-pot mixture were all used as control reactions. The $\mathrm{NzsH} / \mathrm{J} / \mathrm{I}$ reactions with DTT were performed by adding $1 \mathrm{mM}$ DTT in the one-pot system.

Isotopic Labeling Experiments. To verify that the oxygen attached to $\mathrm{C} 4$ of 9 (1-(2-hydroxypropyl)-2-methyl-carbazole3,4(9H)-dione) was derived from water, the $\mathrm{NzsH} / \mathrm{J} / \mathrm{I}$ one-pot reaction was performed by incorporating $15 \%{ }^{18} \mathrm{O}$-water. The labeled compound 9a was obtained with theoretically consistent mass data on the HR-ESIMS analysis. We then scaled up the enzymatic reaction to $50 \mathrm{~mL}$ with a $15 \%$ incorporation ratio of ${ }^{18} \mathrm{O}$-water in order to accumulate 9a for ${ }^{13} \mathrm{C}\left\{{ }^{1} \mathrm{H}\right\}$ NMR spectra analysis. The resulting incorporation ratio was calculated by integrating the peak area of $9 \mathrm{a}$ on the mass chromatogram as follows: ${ }^{18} \mathrm{O}$ incorporation ratio $\%=$ peak area 9a/(peak area 9a + peak area 9) $\times 100$.

Structural Characterization of Reaction Products. The compound 8 (3,5-dihydroxyhexan-2-yl 2-(1H-indol-3-yl) acetate) was isolated and purified from the enzymatic reaction, evaporated to dryness, and resolved in $\mathrm{CD}_{3} \mathrm{OD}$ for structure characterization on NMR analysis. The compound 9 was isolated and purified from the enzymatic reaction, evaporated to dryness, and resolved in DMSO- $d_{6}$ for structure characterization on NMR analysis. The NMR data of the compound was collected on a Bruker Avance $600 \mathrm{MHz}$ NMR spectrometer.

Compound 8: ${ }^{1} \mathrm{H}$ NMR, $\delta_{\mathrm{H}}\left(600 \mathrm{MHz}, \mathrm{CD}_{3} \mathrm{OD}\right), 3.89$ (m, H-1), 1.58 (m, H-2a), 1.36 (m, H-2b), 3.44 (m, H-3), 3.49 (q, H-4), 3.67 (s, H-6), 7.41 (d, H-8), 6.91 (dd, $J=7.7,8.1 \mathrm{~Hz}, \mathrm{H}-9$ ), 6.99 (dd, $J=$ 7.7, 8.0 Hz, H-10), 7.25 (d, $J=8.0 \mathrm{~Hz}, \mathrm{H}-8), 7.06$ (s, H-13), 1.06 (d, $J$ = 6.4 Hz, H-14), 1.09 (d, $J=6.1 \mathrm{~Hz}, \mathrm{H}-15)$; HR-ESIMS $m / z[\mathrm{M}+$ $\mathrm{H}]{ }^{+}$calcd for $\mathrm{C}_{16} \mathrm{H}_{22} \mathrm{NO}_{4}{ }^{+} 292.1543$, found 292.1540.

Compound 9: ${ }^{1} \mathrm{H}$ NMR, $\delta_{\mathrm{H}}\left(600 \mathrm{MHz}, \mathrm{DMSO}-d_{6}\right), 7.83-7.86(\mathrm{~m}$, H-5), 7.50-7.55 (m, H-4), 7.22 (dd, $J=5.6,2.9 \mathrm{~Hz}, \mathrm{H}-6, \mathrm{H}-7$ ), 3.97 (q, $J=6.0 \mathrm{~Hz}, \mathrm{H}-11), 2.79\left(\mathrm{~m}, \mathrm{CH}_{2}\right), 1.93\left(\mathrm{~s}, \mathrm{CH}_{3}\right), 1.24$ (d, $J=6.0$ $\left.\mathrm{Hz}, \mathrm{CH}_{3}\right) ;{ }^{13} \mathrm{C}\left\{{ }^{1} \mathrm{H}\right\} \mathrm{NMR}, \delta_{\mathrm{C}}\left(150 \mathrm{MHz}, \mathrm{DMSO}-d_{6}\right), 183.5$ (CO, C3), 172.54 (CO, C-4), 146.3 (C, C-9a), 139.6 (C, C-1), 136.9 (C, C8a), 134.5 (C, C-2), 125.5 (C, C-4b), 123.9 (C, C-6), 123.7 (C, C-7), 120.0 (C, C-5), 113.3 (C, C-8), 110.7 (C, C-4a), 65.7 (CH, C-11), $37.6\left(\mathrm{CH}_{2}, \mathrm{C}-10\right), 23.5\left(\mathrm{CH}_{3}, \mathrm{C}-12\right), 12.0\left(\mathrm{CH}_{3}, \mathrm{C}-13\right)$; HR-ESIMS $m / z[\mathrm{M}+\mathrm{H}]^{+}$calcd for $\mathrm{C}_{16} \mathrm{H}_{16} \mathrm{NO}_{3}{ }^{+} 270.1125$, found 270.1125 .

Compound 9a: ${ }^{13} \mathrm{C}\left\{{ }^{1} \mathrm{H}\right\}$ NMR, $\delta_{\mathrm{C}}\left(150 \mathrm{MHz}\right.$, DMSO- $\left.d_{6}\right), 184.0$ (CO, C-3), 172.54 and 172.50 (CO and $\left.\mathrm{C}^{18} \mathrm{O}, \mathrm{C}-4\right), 147.1$ (C, C-9a), 140.2 (C, C-1), 137.8 (C, C-8a), 134.6 (C, C-2), 126.0 (C, C-4b), 123.9 (C, C-6), 123.8 (C, C-7), 120.2 (C, C-5), 113.8 (C, C-8), 110.9 (C, C-4a), $66.0(\mathrm{CH}, \mathrm{C}-11), 37.9\left(\mathrm{CH}_{2}, \mathrm{C}-10\right), 23.7$ (CH3, C-12), $12.2\left(\mathrm{CH}_{3}, \mathrm{C}-13\right)$; HR-ESIMS $\mathrm{m} / z[\mathrm{M}+\mathrm{H}]^{+}$calcd for $\mathrm{C}_{16} \mathrm{H}_{16} \mathrm{NO}_{2}{ }^{18} \mathrm{O}^{+}$272.1167, found 272.1166.

Sequence Similarity Network Analysis. The homologues of $\mathrm{NzsH}$, NzsI, and NzsJ from different microorganisms were obtained by blastp search by using $\mathrm{NzsH}$, NzsI, and NzsJ as query sequences, respectively. Adjacent to these $n z s I$-like genes, there are $n z s H$-like and $n z s$-like genes located in some gene clusters (Table S3). The proteins of these $n z s I$-like, $n z s H$-like, and $n z s J$-like genes were selected for further network analysis. The network was constructed by an all-by-all blastp comparison of each sequence against each other sequence and was generated using the EFI-Enzyme Similarity Tool. ${ }^{13}$ Sequence similarity networks were visualized in Cytoscape $3.6 .1^{14}$ to illustrate the distribution of $\mathrm{NzsH}$, NzsI, and NzsJ cluster in microorganisms. The nodes were arranged by using the yFiles organic layout with manual adjustment. Accession numbers were listed in Table S3. The alignment identity of NzsI homologues was visualized with a heat map.

Sequence Alignment Analysis. The multiple sequence alignment of NzsJ, KAS III proteins, KAS III-like proteins, and NzsJ homologues were performed by ClustalX $2^{15}$ and visualized by ESPript 3.0. ${ }^{16}$ To determine conserved motifs in 20 NzsI-like sequences analyzed by blast and cluster mining, MEME ${ }^{12}$ search was performed with the following parameters: $\bmod =$ zoops, nmotifs $=30, \operatorname{minw}=6$, and maxwidth $=50$.

Computational Methods for Theoretical Calculation. HRESIMS data for compound $\mathbf{8}$ indicated a molecular formula of $\mathrm{C}_{16} \mathrm{H}_{21} \mathrm{NO}_{4}$, suggesting 7 degrees of unsaturation in the structure of compound 8. Detailed analysis of ${ }^{1} \mathrm{H},{ }^{13} \mathrm{C}\left\{{ }^{1} \mathrm{H}\right\}$, COSY, HSQC, and HMBC data enabled the construction of the spin systems. A good correlation $\left(r^{2}=0.9984\right)$ of predicted ${ }^{13} \mathrm{C}$ NMR versus experimental ${ }^{13} \mathrm{C}\left\{{ }^{1} \mathrm{H}\right\}$ NMR data suggested the structure is possibly correct. ${ }^{17} \mathrm{ID}$ and 2D NMR data were inputted to the ACD/Laboratories Structure Elucidator and all possible structures calculated within an average difference of $4 \mathrm{ppm}$ difference between calculated and experimental chemical shifts, yielding 48 possible structures. The top 5 calculated structures were calculated by the Structure Elucidator with ${ }^{13} \mathrm{C}$ chemical shift deviations between the experimental and the predicted HOSE-code (dA) ${ }^{18}$ Artificial Neural Net (dN), and Incremental Method. After ranking using the Neural Network Match Factor, the resulted number one candidate is inconsistent with the predicted structure of $\mathbf{8}$. 


\section{ASSOCIATED CONTENT}

\section{S Supporting Information}

The Supporting Information is available free of charge at https://pubs.acs.org/doi/10.1021/acs.joc.9b02688.

Additional tables of primers, strains, and protein accession numbers; NMR, HR-ESIMS, and UV spectra of reactions and products; sequence analysis of NzsJ and NzsI; DFT computation data and parameters (PDF)

\section{AUTHOR INFORMATION}

\section{Corresponding Authors}

*E-mail: h.deng@abdn.ac.uk.

*E-mail: yu_yi@whu.edu.cn.

ORCID

Jioji Tabudravu: 0000-0002-6930-6572

Kwaku Kyeremeh: 0000-0002-3644-9497

Hai Deng: 0000-0002-0047-0622

Yi Yu: 0000-0001-8590-0574

\section{Author Contributions}

OY.L. and L.S. contributed equally to this work.

Notes

The authors declare no competing financial interest.

\section{ACKNOWLEDGMENTS}

We thank the National Key Research and Development Program of China (2018YFA0900400), the National Natural Science Foundation of China (31570033, 31811530299, and 31870035 to Y.Y.), the Royal Society-NSFC Newton Mobility

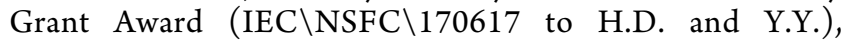
Biotechnology and Biological Sciences Research Council UK (H.D., K.R., and L.T., BB/R00479X/1), the Elphinstone Scholarship of University of Aberdeen (Q.F.), Leverhulme Trust-Royal Society Africa award (AA090088), and Medical Research Council UK Africa Future leader (K.K. and H.D., $\mathrm{MR} / \mathrm{S} 00520 \mathrm{X} / 1$ ) for financial support.

\section{REFERENCES}

(1) Huang, S.; Elsayed, S. S.; Lv, M.; Tabudravu, J.; Rateb, M. E.; Gyampoh, R.; Kyeremeh, K.; Ebel, R.; Jaspars, M.; Deng, Z.; Yu, Y.; Deng, $\mathrm{H}$. Biosynthesis of neocarazostatin A reveals the sequential carbazole prenylation and hydroxylation in the tailoring steps. Chem. Biol. 2015, 22, 1633-1642.

(2) Kato, S.; Shindo, K.; Kataoka, Y.; Yamagishi, Y.; Mochizuki, J. Studies on free radical scavenging substances from microorganisms. $J$. Antibiot. 1991, 44, 903-907.

(3) Su, L.; Lv, M.; Kyeremeh, K.; Deng, Z.; Deng, H.; Yu, Y. A ThDP-dependent enzymatic carboligation reaction involved in Neocarazostatin A tricyclic carbazole formation. Org. Biomol. Chem. 2016, 14, 8679-8684.

(4) Su, L.; Zhang, R.; Kyeremeh, K.; Deng, Z.; Deng, H.; Yu, Y. Dissection of the neocarazostatin: a C4 alkyl side chain biosynthesis by in vitro reconstitution. Org. Biomol. Chem. 2017, 15, 3843-3848.

(5) Kobayashi, M.; Tomita, T.; Shin-Ya, K.; Nishiyama, M.; Kuzuyama, T. Discovery of unprecedented carbazole alkaloidsynthesizing machinery in Streptomyces. Angew. Chem., Int. Ed. 2019, 58, 13349-13353.

(6) Prasad, G.; Amoroso, J. W.; Borketey, L. S.; Schnarr, N. A. Nactivated $\beta$-lactams as versatile reagents for acyl carrier protein labeling. Org. Biomol. Chem. 2012, 10, 1992-2002.

(7) Storm, P. A.; Townsend, C. A. In trans hydrolysis of carrier protein-bound acyl intermediates by CitA during citrinin biosynthesis. Chem. Commun. 2018, 54, 50-53.
(8) Kawamura, M.; Kamo, S.; Azuma, S.; Kubo, K.; Sasamori, T.; Tokitoh, N.; Kuramochi, K.; Tsubaki, K. Skeletal rearrangements of polycyclic $\alpha$-ketols. Org. Lett. 2017, 19, 301-303.

(9) Proteau, P. J.; Li, Y.; Chen, J.; Williamson, R. T.; Gould, S. J.; Laufer, R. S.; Dmitrienko, G. I. Isoprekinamycin is a diazobenzo[a]fluorene rather than a diazobenzo[b]fluorene. J. Am. Chem. Soc. 2000, $122,8325-8326$.

(10) Diakur, J.; Nakashima, T. T.; Vederas, J. C. Magnitudes of ${ }^{18} \mathrm{O}$ isotope shifts in ${ }^{13} \mathrm{C}$ nuclear magnetic resonance spectra of ketones and alcohols. Can. J. Chem. 1980, 58, 1311-1315.

(11) Intaraudom, C.; Rachtawee, P.; Suvannakad, R.; Pittayakhajonwut, P. Antimalarial and antituberculosis substances from Streptomyces sp BCC26924. Tetrahedron 2011, 67, 7593-7597.

(12) Bailey, T. L.; Johnson, J.; Grant, C. E.; Noble, W. S. The MEME Suite. Nucleic Acids Res. 2015, 43, W39-49.

(13) Gerlt, J. A.; Bouvier, J. T.; Davidson, D. B.; Imker, H. J.; Sadkhin, B.; Slater, D. R.; Whalen, K. L. Enzyme Function InitiativeEnzyme Similarity Tool (EFI-EST): A web tool for generating protein sequence similarity networks. Biochim. Biophys. Acta, Proteins Proteomics 2015, 1854 (8), 1019-1037.

(14) Shannon, P.; Markiel, A.; Ozier, O.; Baliga, N. S.; Wang, J. T.; Ramage, D.; Amin, N.; Schwikowski, B.; Ideker, T. Cytoscape: a software environment for integrated models of biomolecular interaction networks. Genome Res. 2003, 13 (11), 2498-2504.

(15) Larkin, M. A.; Blackshields, G.; Brown, N. P.; Chenna, R.; McGettigan, P. A.; McWilliam, H.; Valentin, F.; Wallace, I. M.; Wilm, A.; Lopez, R.; Thompson, J. D.; Gibson, T. J.; Higgins, D. G. Clustal W and clustal X version 2.0. Bioinformatics 2007, 23 (21), 29472948.

(16) Robert, X.; Gouet, P. Deciphering key features in protein structures with the new ENDscript server. Nucleic Acids Res. 2014, 42 (W1), W320-W324.

(17) Williams, A. J.; Elyashberg, M. E.; Blinov, K. A.; Lankin, D. C.; Martin, G. E.; Reynolds, W. F.; Porco, J. A.; Singleton, C. A.; Su, S. Applying computer-assisted structure elucidation algorithms for the purpose of structure validation: Revisiting the NMR assignments of hexacyclinol. J. Nat. Prod. 2008, 71 (4), 581-588.

(18) Bremser, W. Hose - a novel substructure code. Anal. Chim. Acta 1978, 103 (4), 355-365. 